\title{
Numerical Investigation of Wingtip Vortex Attenuation Using a Tip-Chipped Wing*
}

\author{
Nahyeon $\mathrm{RoH},{ }^{1)}$ Sejong $\mathrm{OH},{ }^{1)}$ and Kwanjung $\mathrm{YEE}^{2) \dagger}$ \\ ${ }^{1)}$ Department of Aerospace Engineering, Pusan National University, Pusan 46241, Korea \\ ${ }^{2)}$ Department of Mechanical and Aerospace Engineering, Seoul National University, Seoul 08826, Korea
}

\begin{abstract}
The attenuation of wingtip/blade vortices has been one of the primary concerns in aviation safety and blade-vortex noise. In an effort to effectively dissipate the vortex intensity, a trailing-edge (TE) chipped wing concept was suggested. In this study, to exploit the potential of the chipped wingtip concept for alleviating the tip vortex, a series of numerical simulations are conducted. The numerical simulations are performed using open source code, OpenFOAM. The wake structures are measured for different wingtip shapes. The chip depth and location are varied to evaluate the vortex alleviation rate and aerodynamic characteristics. As a result of numerical simulation, it is confirmed that the vortex dissipation rate of the tip-chipped wing is higher than that of a TE-chipped wing. For the tip-chipped wing, the counter-rotating vortex is found to be strong enough to weaken the primary vortex. The dissipation rate increases as the location of the chip gets closer to the leading-edge, and as the depth of the chip increases. A trade-off relationship between vortex alleviation and an increase in drag is confirmed. For example, the MID D3 attenuates the strength of the wingtip vortex by $51 \%$, while the drag is increased within $5 \%$.
\end{abstract}

Key Words: Tip-chipped Wingtip Shape, Computational Fluid Dynamics, Trailing Vortex Attenuation

\section{Nomenclature}

$x$ : stream-wise coordinate

$y$ : swirl-wise coordinate

$z$ : span-wise coordinate

$u$ : stream-wise velocity

$v$ : swirl-wise velocity

$w$ : span-wise velocity

$U_{\infty}$ : freestream velocity

$V_{\theta}$ : swirl velocity

$c$ : chord length of wing

$b$ : span length of wing

$C_{L}:$ lift coefficient

$C_{D}$ : drag coefficient

$L / D$ : lift-to-drag ratio

$\omega$ : vorticity magnitude

$v_{p p}$ : peak-to-peak swirl velocity

Subscripts

$\infty$ : infinity

pp: peak-to-peak

\section{Introduction}

\subsection{Motivation}

At the wingtip of aircraft and blade tip of rotors, a pair of strong vortices is generated. This vortex is produced due to the pressure difference between the upper and lower surfaces of the wing.

For years, the effect of wingtip vortices on aircraft has

(C) 2017 The Japan Society for Aeronautical and Space Sciences

*Received 10 November 2015; final revision received 26 January 2017; accepted for publication 27 January 2017.

†Corresponding author, kjyee@snu.ac.kr been a serious aviation safety issue in the airport operation. When a small plane passes through the trailing vortex of a larger aircraft (Fig. 1), ${ }^{1)}$ the following plane experiences a substantial rolling moment remove due to the swirl of the vortex. If this happens during landing or takeoff, the pilot has no time to regain the control of aircraft due to its low altitude and crashes. Since the vortex wakes of the leading aircraft are persistent and can be hazardous, the following aircraft must delay its arrival until the vortex wakes have decayed to a harmless level. This takes more than three minutes considering conventional aircraft operation. ${ }^{2)}$ Separation time intervals can be shortened by reducing the intensity of the vortices created by the leading aircraft, thereby leading to the enhanced efficiency of airport operation.

For the case of helicopter operation, there is a potential for significant interaction between the vortex created by the leading rotor blade and the trailing rotor blade that subsequently passes through it. These interactions between vortex-vortex and vortex-blade become a source of unsteady aerodynamic forces, and can be a significant source of blade-vortex interaction (BVI) noise (Fig. 2). ${ }^{3)}$ This noise is loud and impulsive in nature, which is significant both for military detection and community annoyance. ${ }^{4)}$ It is known that small changes in the structure of the tip vortices can substantially reduce BVI noise. Hence, there is great demand to develop simple yet effective devices for reducing the vortex strength.

\subsection{Previous study}

To comprehend the wake structure behind a wing, many studies have been investigated both theoretically ${ }^{5-7)}$ and experimentally. ${ }^{8-12)}$ Rayleigh $^{5)}$ developed a formal theory for the stability of swirling flows. He studied the effects of axisymmetric disturbances on vortices. Batchelor ${ }^{6)}$ derived an 


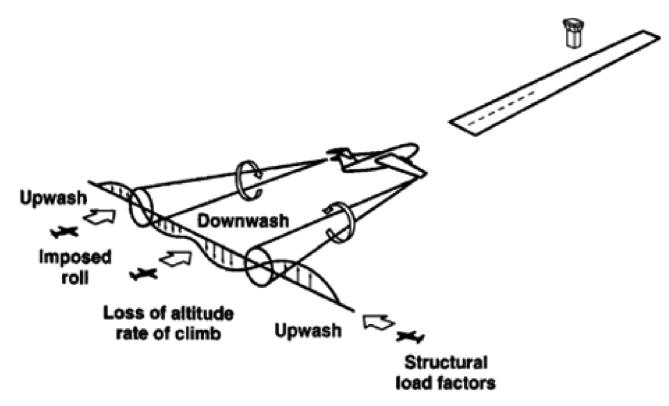

Fig. 1. Possible encounters with lift-generated wake for the following aircraft. ${ }^{1)}$

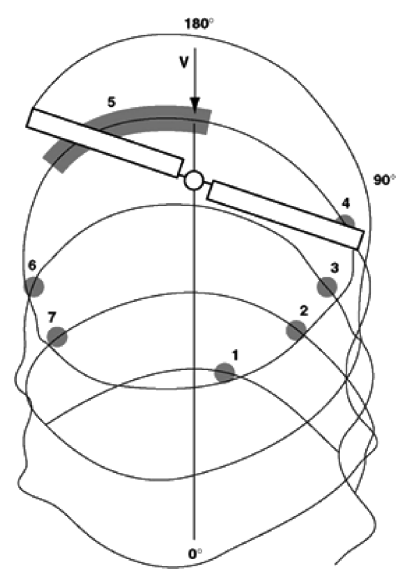

Fig. 2. Blade-Vortex interactions during descending flight (numbers 1-7 indicate blade-vortex interactions). ${ }^{3 \text { ) }}$

asymptotic solution for trailing vortices in the far wake of a lifting surface. Saffman ${ }^{7)}$ examined the effects of the Reynolds number on the decay of turbulent line vortices. Bleviss ${ }^{8)}$ conducted an experimental study on the physical characteristics of vortices. He suggested the amount of distance and time required for the vortices to decay to a harmless level. Rossow $^{9)}$ found the discrete boundaries, which distinguish merging from non-merging situations. Devenport et al. ${ }^{10)}$ made an extensive set of hot-wire measurements on a wing with a NACA0012 airfoil. They performed a detailed analysis to reveal the flow structure in the core region. Chow et al. ${ }^{11)}$ conducted experimental wind tests to investigate the rollup of a wingtip vortex. They found that the turbulence decayed quickly with stream-wise distance because of the stabilizing effect of the nearly solid body rotation of the vortex core mean flow.

Rossow $^{12)}$ suggested that the opposite sign of vortex is effective to alleviate the primary vortex. On the basis of his theory, trailing vortex wake alleviation studies have been conducted over the past four decades; wingtip blowing, ${ }^{13}$ ) wingtip turbine, ${ }^{14)}$ wing fins, ${ }^{15)}$ gurney flap, ${ }^{16)}$ active flap, ${ }^{17)}$ slotted tip ${ }^{18)}$ and ogee tip. ${ }^{19)}$

Recently, Jin et al. ${ }^{20)}$ proposed a TE-chipped wing that has a rectangular hole at the trailing-edge. Through the TEchipped wing, the wingtip vortex is alleviated as the reinteraction between the primary vortex generated by the wingtip and sub-vortices generated at the chip.

\subsection{Research objectives}

The goal of this study is to exploit the potential of the chipped wingtip concept for alleviating the tip vortex based on Jin's previous study. ${ }^{20)}$ Jin et al. confirmed that the vortex strength is reduced by more than $40 \%$ over the TE-chipped wing. However, at the TE-chipped wing, the vortices occurred at wingtip and the chip begin to mix at the trailingedge. Thus, it was hard to dissipate the fully developed wingtip vortex. Therefore, in this study, wings that interfere during the generation process of the wingtip vortex are designed. The wing that has a chip at the wingtip is named "tip-chipped wing." To evaluate the effects of the tip chip shape, a series of parametric studies are conducted with regard to the chip location and depth.

\section{Numerical Methods}

\subsection{Solution procedures}

In the present study, a series of computations are carried out using open source code, OpenFOAM (full Navier-Stokes equation). The 1st-order Euler scheme for the time integration and 2nd-order Gauss linear upwind scheme for convective terms are used. A PIMPLE algorithm that combines the characteristics of the Semi-Implicit Method for Pressure Linked Equation (SIMPLE) algorithm with the Pressure Implicit with Splitting Operator (PISO) algorithm was used. To validate against the Devenport's experimental results, ${ }^{10)}$ the Reynolds number based on airfoil chord is kept at 530,000 and the angle of attack is kept at $5^{\circ}$.

\subsection{Turbulence model}

A realizable $k-\varepsilon$ turbulence model was used. The Standard $k-\varepsilon$ turbulence model performs quite well for boundary layer flows, but not for flows with a high mean shear rate or massive separation. ${ }^{21)}$ The realizable $k$ - $\varepsilon$ model contains a new formulation for turbulent viscosity and a new transport equation for the dissipation rate, $\varepsilon$. The realizable $k-\varepsilon$ model satisfies certain mathematical constraints for Reynolds stress, and is consistent with the physics of turbulent flow. ${ }^{22)}$

\subsection{Computational domain}

The computational domain included a wing that was used for the experiments of Devenport et al. ${ }^{10)}$ The baseline wing is a rectangular planform with truncated a tip and NACA0012 airfoil having a chord of $0.203 \mathrm{~m}$. The half-wing span was $0.879 \mathrm{~m}$, and the AR was 8.66. Hereafter, the airfoil chord is $c$, and wing span is $b$. As shown in Fig. 3, the complete domain encompasses the region of $-30 \leq x / c \leq$ $30,-30 \leq y / c \leq 30$ and $0 \leq z / c \leq 5 \times b / 2$, with a grid of $71 \times 121 \times 123$, in the stream-wise, surface normal, and span-wise directions, respectively. The origin of the coordinate is located at the airfoil trailing-edge. A wall function is adopted for the sub-layer. Therefore, a viscous grid spacing of $1 \times 10^{-3} c$ was specified on the wing surface with the aim of setting $y^{+} \cong 30$. A C-H topology was used for the grid geometry. To accurately resolve the vortex structure, downstream of the wingtip region was generated by clustering an equally spaced fine grid (Fig. 4). 


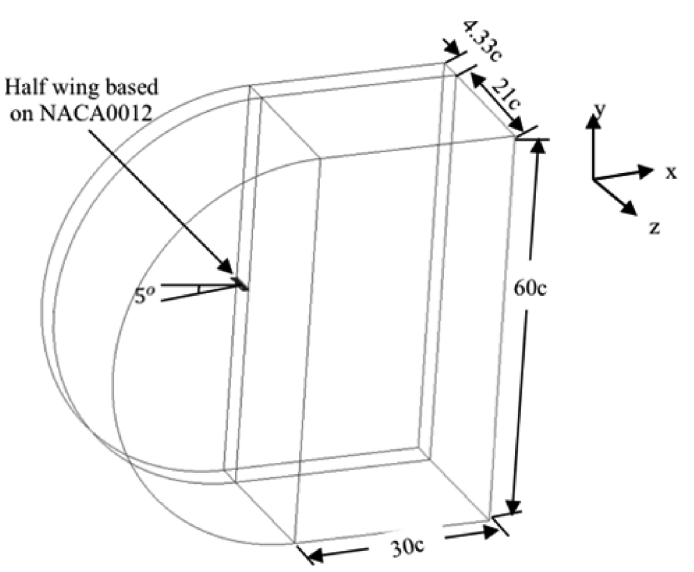

Fig. 3. Computational domain.

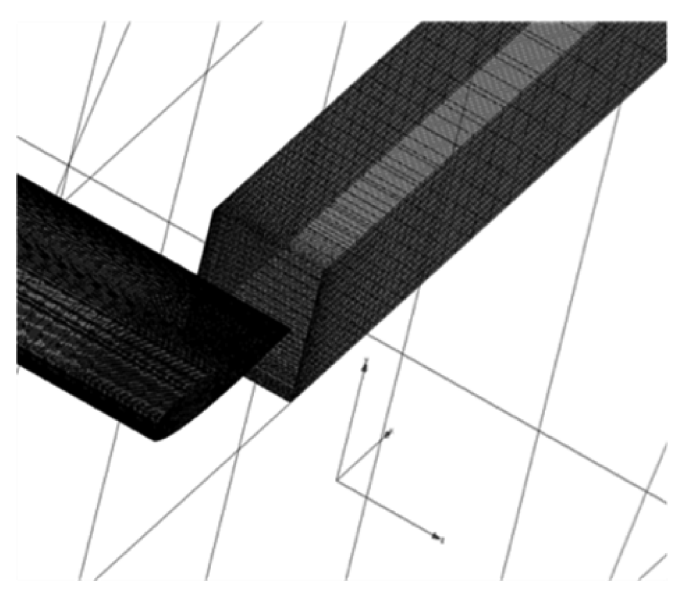

Fig. 4. Grid clustering of wingtip region.

\subsection{Tip-chipped wing shape for alleviation}

The TE-chipped shape ${ }^{20)}$ and tip-chipped shapes are defined as shown in Fig. 5. In order to compare with the results of Jin et al. ${ }^{20)}$ four shapes that have the same chip area are examined. The location of the chip is varied with respect to chord-wise location, such as front (FR), middle (MID) and rear (RE) of the wingtip. The chip areas of all the shapes is less than $1.3 \%$ of the wing.

The variation in chip depth is shown in Fig. 5. The FR chipped shape is varied from D1 to D4. The MID and RE chipped shapes are varied from D2 to D4. Variation from D1 to D4 means that chip depth is changed from 0.1 to 0.4 chord.

For the TE-chipped shape, spacing between the wingtip and the chip is 0.15 chord. To maintain consistency with Jin's study, ${ }^{20)}$ the FR/RE chip is located at 0.15 chord from the leading and trailing edges, respectively. The chip center of the MID chip is 0.5 chord from the leading-edge. A summary of chip shapes is presented in Fig. 6.

\section{Numerical Validation}

\subsection{Validation for solver and grid}

Validation studies are conducted to verify the computational grid, solver and turbulence model. The results are com-

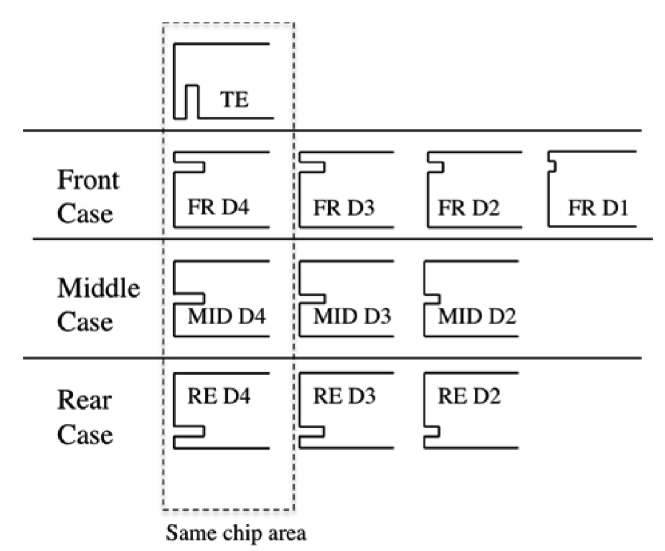

Fig. 5. Definition of TE-chipped shape and tip-chipped shapes.

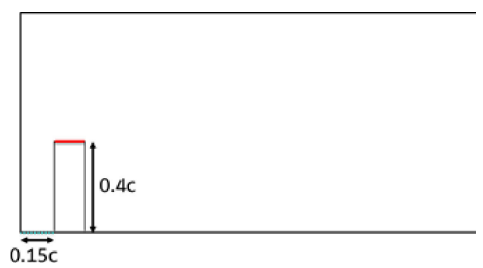

(a) TE-chipped shape

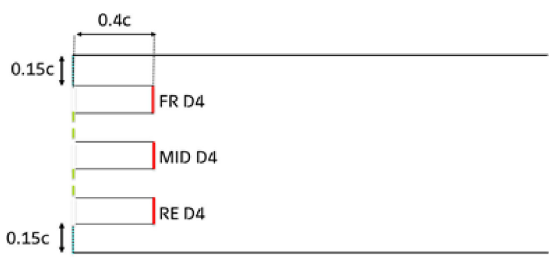

(b) Tip-chipped shape

Fig. 6. Summary of TE-chipped shape and tip-chipped shapes with location variation.

Table 1. Grid test for numerical validation.

\begin{tabular}{cllcc}
\hline Grid & Total cells & Spacing & Time & $V_{\theta} / U_{\infty}$ at $10 c$ \\
\hline Grid 1 & $4,755,520$ & 0.002 & $15 \mathrm{~h}$ & 0.2345 \\
Grid 2 & $5,182,500$ & 0.001 & $18 \mathrm{~h}$ & 0.2705 \\
Grid 3 & $9,138,075$ & 0.0005 & $28 \mathrm{~h}$ & 0.2758 \\
\hline
\end{tabular}

pared with the experimental results of Devenport et al. ${ }^{10)}$ and numerical results of Jin et al. ${ }^{20)}$ Jin et al. employed a commercial solver, ANSYS FLUENT and the Reynolds stress model (RSM). The RSM, a seven-equation model, is the most elaborated type of turbulence model that ANSYS FLUENT provides. The present results are obtained using OpenFOAM with two equation model, realizable $k-\varepsilon$.

A grid sensitivity study was conducted using the three grids and varying resolutions. The number of total cells, grid spacing, time and results are summarized in Table 1. The stream-wise grid distribution was not changed. Figure 7 compares swirl velocities through the vortex center at 10 chords downstream of the trailing-edge. As can be seen in Fig. 7, Grid 1 indicates severely under-predicted swirl velocity with $15 \%$ error. The swirl velocity profiles of both Grid 2 and Grid 3 show reasonable results compared to the experiments, having a $3 \%$ error in peak swirl velocity; whereas, the Grid 3 requires more computational time than Grid 2, ap- 
proximately 1.5 times more than. Therefore, in this study, Grid 2 is utilized for analysis.

On the other hand, the core radius is not predicted well when compared to using RSM. This comes from the fundamental limitation of turbulence model accuracy between RSM and realizable $k-\varepsilon$. Nevertheless, this study primarily focuses on the process and tendency of vortex interactiondissipation, and the vortex alleviation effects are obtained by peak swirl velocity. Accordingly, it is assumed to be an acceptable level of error.

In addition, the computation resources and time for convergence in OpenFOAM with realizable $k-\varepsilon$ is almost $18 \mathrm{~h}$ using seven nodes of a Xenon $3.2 \mathrm{GHz}$ processor. However, in the case of FLUENT with RSM, more than $70 \mathrm{~h}$ is required using seven nodes of an AMD 4.0 GHz processor. Thus, it is confirmed that realizable $k-\varepsilon$ in OpenFOAM offers a similar level of accuracy to RSM in FLUENT doing so requiring less computation resources and time.

\subsection{Validation of turbulence model}

The $k$ - $\omega$ SST turbulence model is used for comparison because it is known to have a superior capability for capturing massive separation. ${ }^{23)}$ The swirl velocity distribution at 10 chords downstream from the trailing-edge of the wing is compared. As shown in Fig. 7, the realizable $k-\varepsilon$ turbulence model provides better agreement with the experimental data than the $k-\omega$ SST turbulence model. This is attributed to a characteristic of the $k-\omega$ SST turbulence model in which the standard $k$ - $\varepsilon$ turbulence model is used in the flow field. The realizable $k-\varepsilon$ turbulence model ensures the 'realizable'

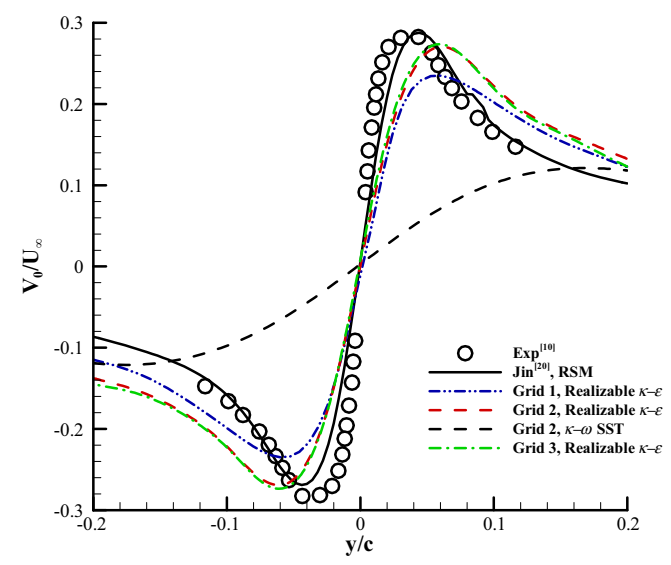

Fig. 7. Swirl velocity distribution at $x / c=10$. outcome by using a formulation for $c_{\mu}$, coefficient of turbulence viscosity, which is a constant value in the standard $k-\varepsilon$ turbulence model. It can be seen that the vortex is preserved quite well with the realizable $k-\varepsilon$ turbulence model.

\section{Results and Discussion}

\subsection{Comparison between TE-chipped shape and tip- chipped shape}

The interacting and merging processes of the vortices are greatly affected by the distance, strength and direction between the vortices. ${ }^{9)}$ To observe the wingtip vortex formation and diffusion processes, axial vortices are examined. FR D4, which will be shown to be the most effective shape in wingtip vortex mitigation, is compared with the TE-chipped shape.

The vorticity magnitude contours around the wing surface and near-field are illustrated in Fig. 8. As seen in Fig. 8(a), for the TE chip the primary tip vortex is fully developed without any interference, while for the tip chip, the primary tip vortex shows interference during formation, as shown in Fig. 8(b). For both the TE and tip chips, the chip generates the sub-vortices.

The axial vorticity contours from $x / c=0.0$ to $x / c=3.0$ with an interval of 0.5 chord are shown in Fig. 9. For both the TE and tip chips, the first sub-vortex rotates in the opposite direction of the primary vortex and the second sub-vortex rotates in the same direction as the primary vortex. For the TE chip, since the chip is in the chord-wise direction, the sub-vortices are generated in proximity. Therefore, two sub-vortices with opposite signs are mixed in near-field. Due to this interaction, the strength of the first sub-vortex, which plays a key role for attenuation of the primary vortex, is weakened. Subsequently, this weakened first sub-vortex is quickly absorbed into the primary vortex. For the tip chip, the first sub-vortex is integrated into the primary vortex without any other dissipation process. Because the tip chip is in the span-wise direction, the sub-vortices are generated at a distance. Thus, the interaction process between sub-vortices is nearly negligible. As a result, the primary vortex is weakened more than that of the TE chip. The phenomena described above can be explained by comparing the vorticity contours immediately after the merging of the first sub-vortex. The first sub-vortices for the TE and tip chips are almost mixed with the primary vortex at $x / c=1.0$ and $x / c=3.0$,

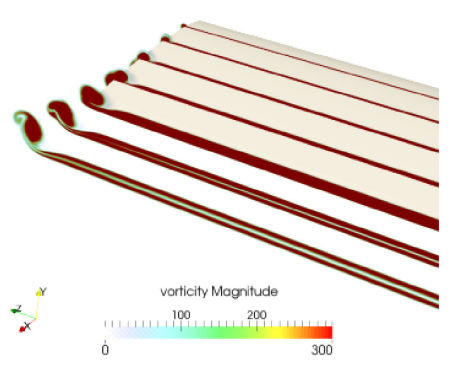

(a) Baseline wing

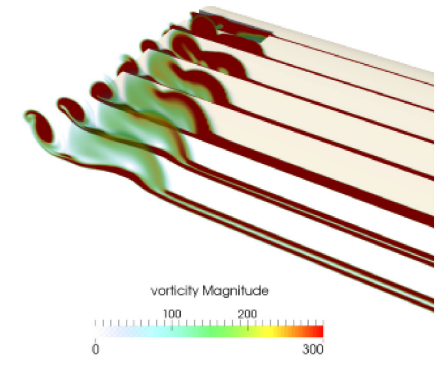

(b) TE-chipped wing

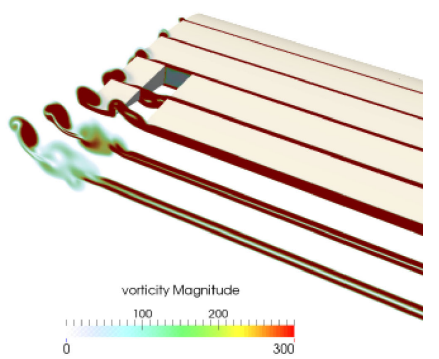

(c) Tip-chipped wing

Fig. 8. Perspective view of vorticity magnitude along the wing surface. 


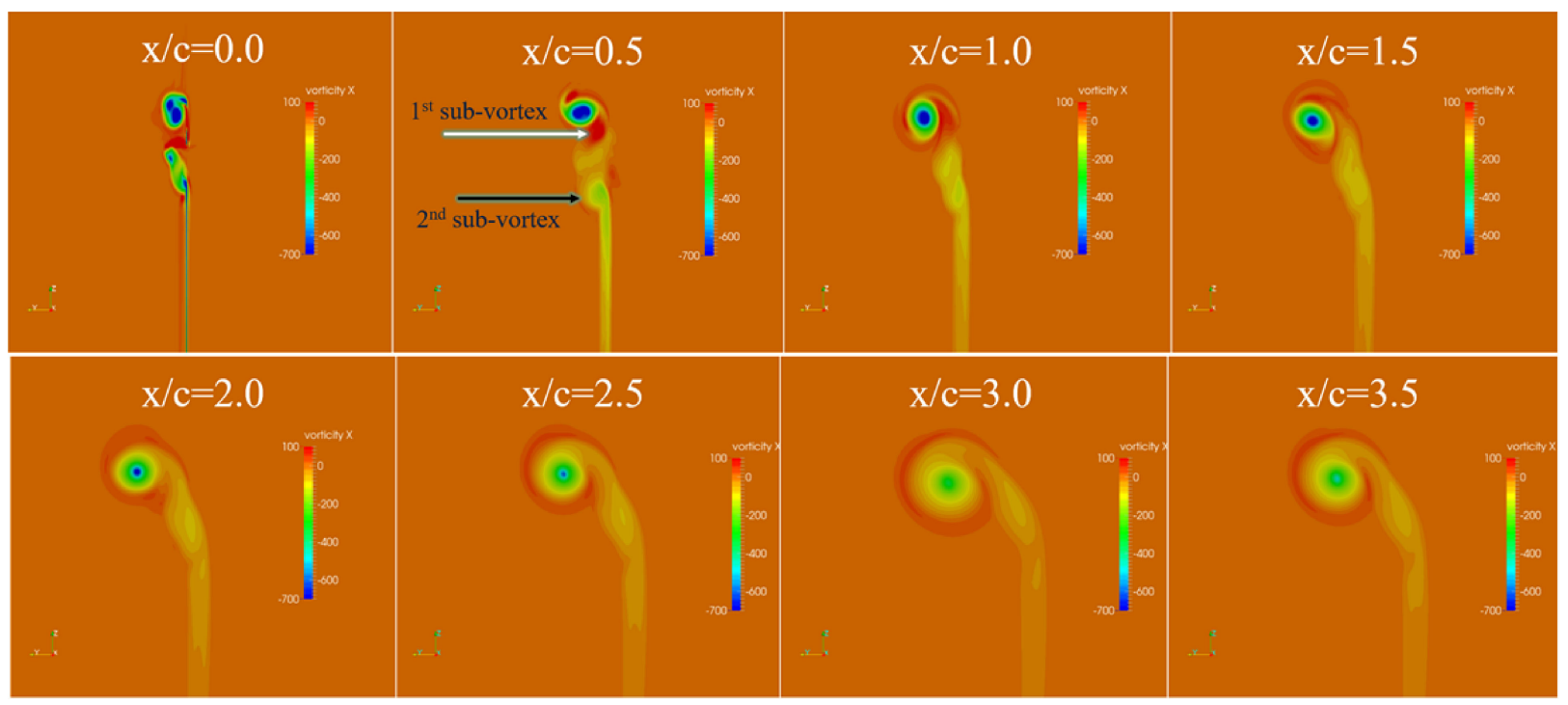

(a) TE chip

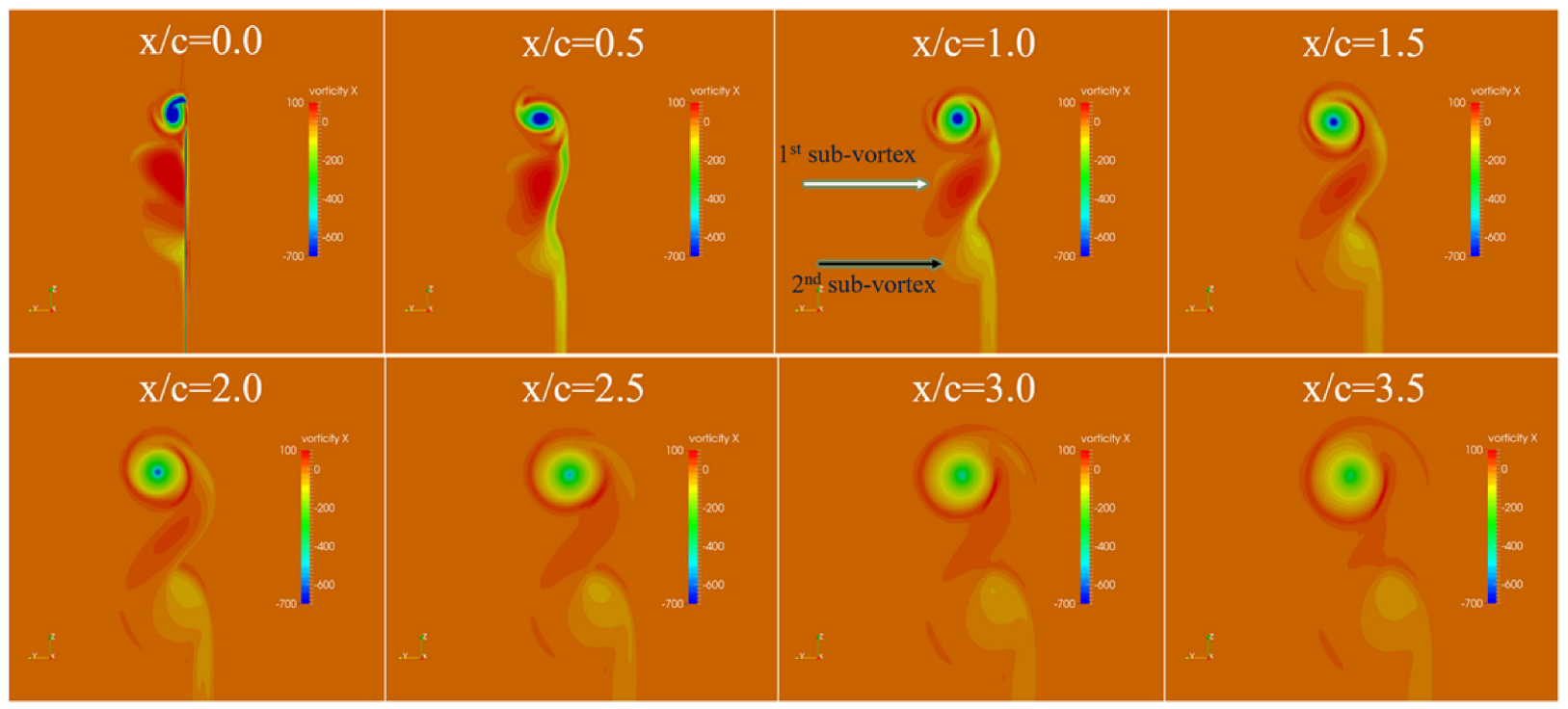

(b) Tip chip

Fig. 9. Axial vorticity contour at near-field.

respectively. At each location, it is obvious that the primary vortex of the tip chip is weaker.

Figure 10 shows the vorticity magnitude contours at the far-field. The data is extracted from $x / c=5.0$ to $x / c=20$ at intervals of 5 chords. For the case of the TE chip, the tip vortex rolls up into a coherent form about 5 chords downstream of the trailing-edge. After that, the wingtip vortex is dissipated smoothly in the stream-wise direction. On the other hand, the vortex structure for the tip chip is not completely axisymmetric, even at $x / c=20$, since the second sub-vortex is still merging. This means that the wingtip vortex is still unstable and additional dissipation will occur, providing further attenuation of the wingtip vortex. Thus, the vortex attenuation effect of the tip chip is more effective than the TE chip.

Next, the important vortex parameters, such as maximum vorticity and swirl velocity, are examined. Figure 11 shows a comparison of the vorticity magnitude and normalized swirl velocity $\left(v / U_{\infty}\right)$ at 20 chords downstream behind the baseline wing, TE chip and tip chip. The maximum vorticity magnitude is reduced by $70.1 \%$ and $86.1 \%$ in the TE chip and tip chip, respectively, compared to the plain wing. The peak swirl velocity is mitigated by $55.4 \%$ and $45.3 \%$, respectively. Additionally, the core radius, defined as half the distance between the peak-to-peak swirl velocity, is increased $154 \%$ and $329 \%$ respectively compared to that of the baseline wing. Thus, it can be seen that the tip chip is more effective in alleviating the wingtip vortex strength than the TE chip.

\subsection{Effects of chip location}

The effects of the chip location are examined in this section. The four shapes, which have the same chip area with different locations, are analyzed. The four different TEchipped shapes, FR D4, MID D4 and RE D4, are used.

\subsubsection{Peak vorticity magnitude}

Figure 12 compares the peak vorticity magnitude with the baseline shape, TE chip and tip chip. The vorticity magnitude 


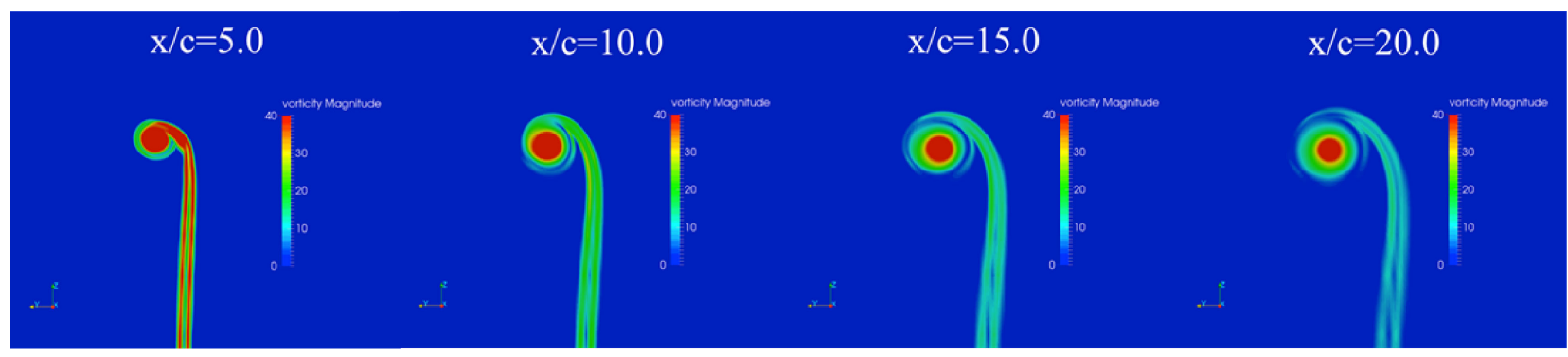

(a) TE chip

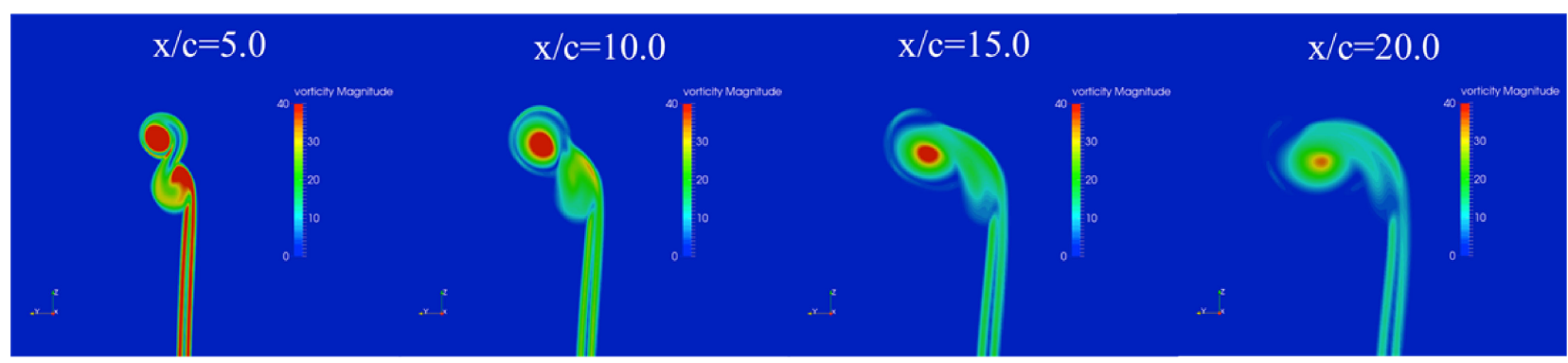

(b) Tip chip

Fig. 10. Vorticity magnitude contour at far-field.
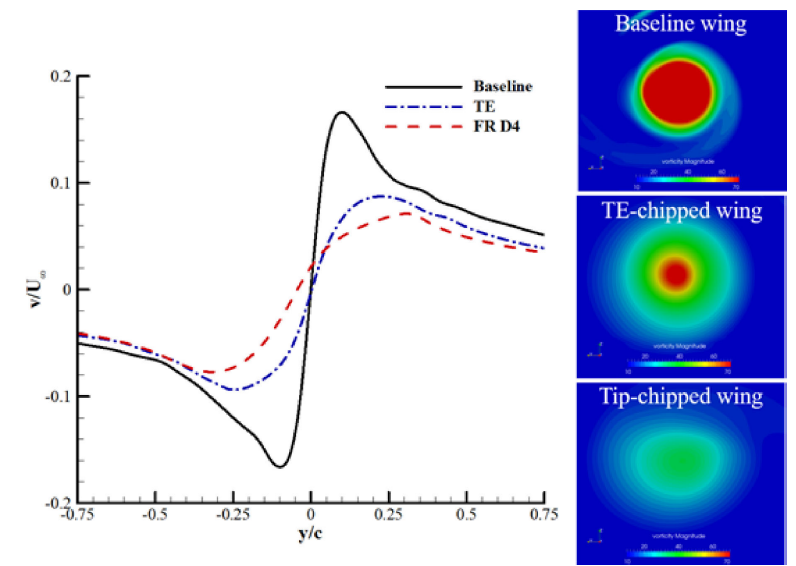

Fig. 11. Vorticity magnitude contour and swirl velocity distribution due to the chip location at $x / c=20$.

decreases for all of chip shapes when compared to the baseline. It also shows that, for the given chip area, the tip chip is more effective in reducing the peak vorticity than the TE chip.

Among the chipped shapes, the initial vorticity magnitude of the TE chip is the highest and lowest for RE D4. The initial vortex strength of the TE chip appears strongest because the primary vortex is fully developed. The initial vortex strength of RE D4 appears the weakest because the primary vortex is disturbed most recently. When the chip moves from the leading-edge to the trailing-edge, the reduction rate of vorticity magnitude decreases. For the case of FR D4, the vorticity magnitude is reduced by $86.1 \%$.

\subsubsection{Velocity distributions}

Figure 13 compares the peak swirl velocities with the baseline shape, TE chip and tip chip. The TE chip and RE D4 have similar reduction rates for peak swirl velocity. FR D4 has the highest reduction rate for peak swirl velocity.

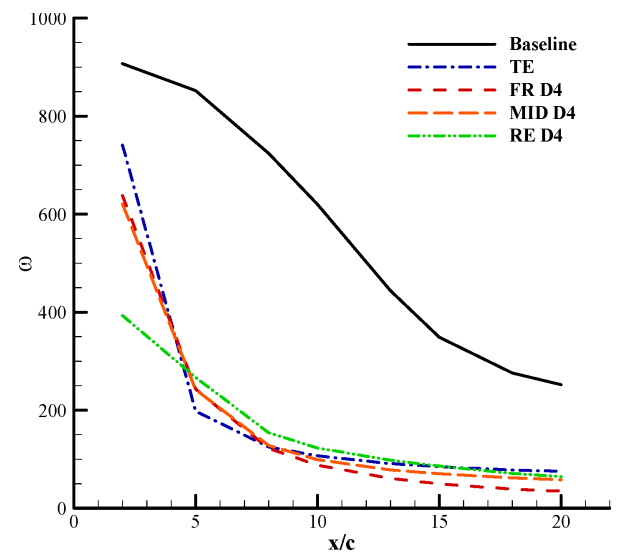

Fig. 12. Peak vorticity magnitude due to the chip location.

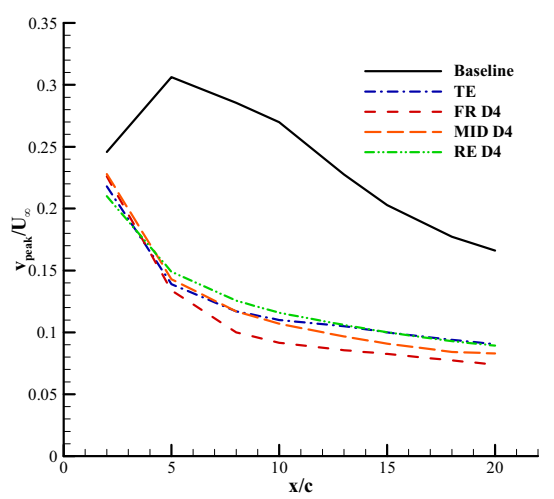

Fig. 13. Peak swirl velocity due to the chip location.

The baseline shape, TE chip and tip chip swirl velocity distributions are shown in Fig. 14. The velocity distribution is plotted at $x / c=20$ to consider the trade-off relationship between limit of computational resources and development of vortices. The TE chip and RE D4 show similar distribu- 


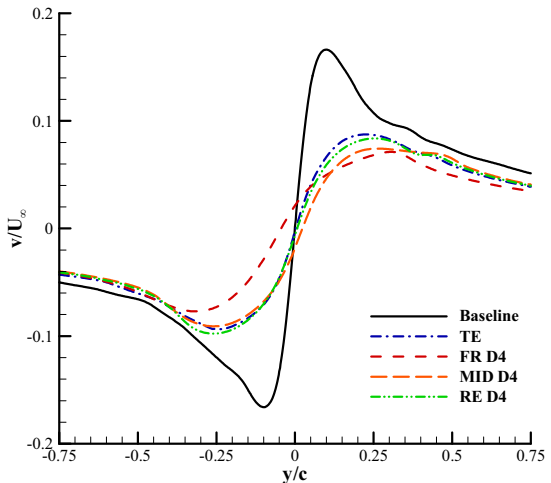

Fig. 14. Swirl velocity distribution due to the chip location at $x / c=20$.

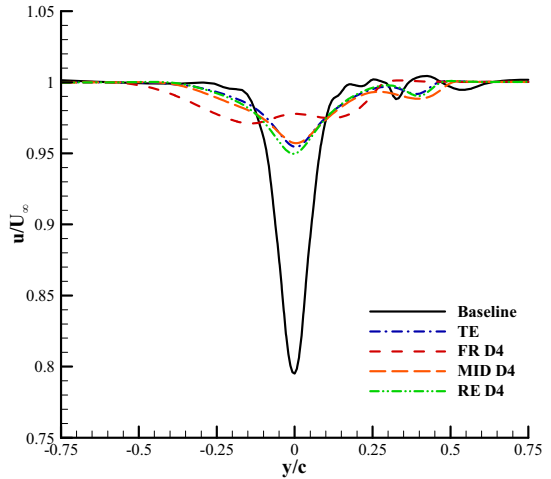

Fig. 15. Axial velocity distribution due to the chip location at $x / c=20$.

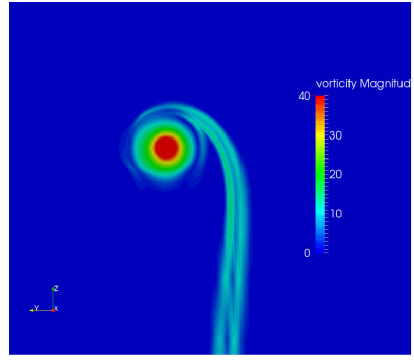

(a) TE chip

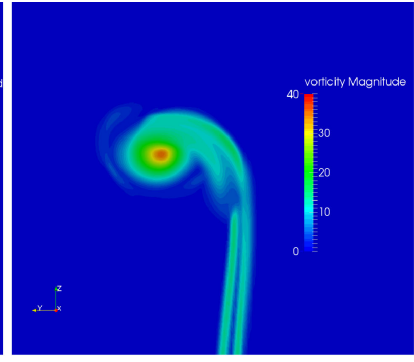

(b) FR chip

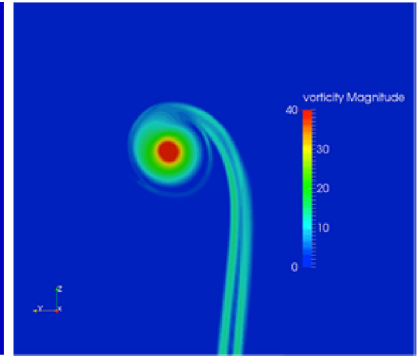

(c) MID chip

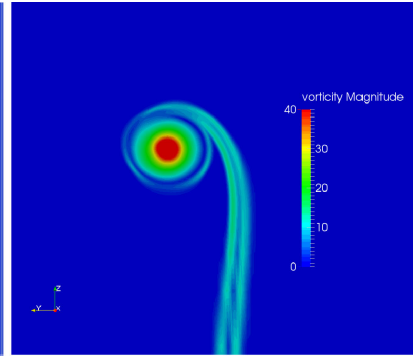

(d) RE chip

Fig. 16. Vorticity magnitude contour at $x / c=20$.

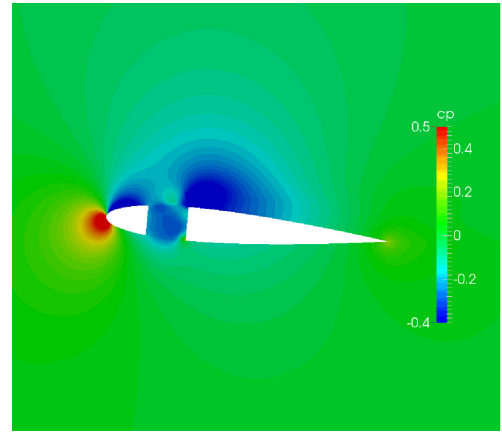

(a) FR chip

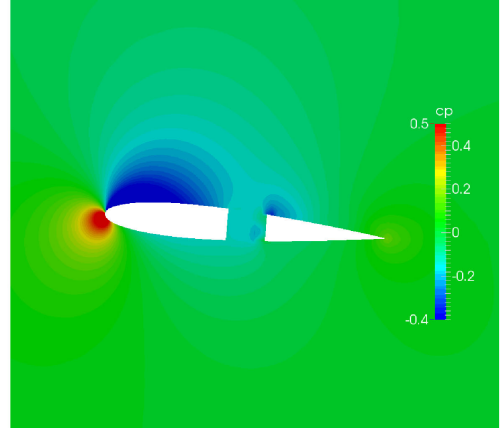

(b) MID chip

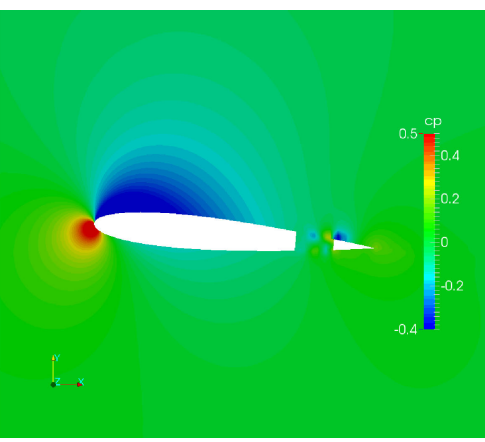

(c) RE chip

Fig. 17. Pressure coefficient contour in the span-wise direction at the chip center.

tions, and peak values are reduced by $45 \%$. FR D4 shows a minimum peak value reduced by $55.4 \%$.

Since the vortex generated by the aircraft remains until 250 chords downstream, it is necessary to study the vortex reduction effects generated by the chipped shapes until far downstream. To this end, the axial velocity distribution needs to be examined. Because the axial velocity deficit will cause radial transport of the angular momentum, ${ }^{24)}$ it can be expected to diminish the swirl velocity at a faster rate.

In Fig. 15, axial velocity distribution at $x / c=20$ for the tip chip is shown compared to the results of the baseline shape and TE chip. An axial velocity deficit is observed for all of the chipped shapes. From this figure, it is apparent that axial velocity distributions have a single peak value in every chip shape except FR D4. These results indicate that the primary vortex and sub-vortices are almost completely merged into a single vortex. Meanwhile, FR D4 has two peaks, which implies that the sub-vortex generated by the chip still remains at 20 chord downstream (Fig. 16). Hence, it is expected that FR D4 has a greater rate of vortex alleviation than the other cases even after 20 chords.

\subsubsection{Pressure distribution}

Through the results of the vortex parameters, it is concluded that FR D4 is the most effective shape for vortex reduction. For wings with an angle of attack, pressure on the upper surface is lower than that on the lower surface. When the chip is located close to the leading-edge, the chip experiences a higher pressure difference, as can be seen in Fig. 17. It is assumed that the high pressure difference brings about strong sub-vortices. Figure 17 shows the pressure contour at the chip centerline. The case of FR D4 yields a higher pressure difference across the chip, providing strong interference effects on wingtip vortex generation. 


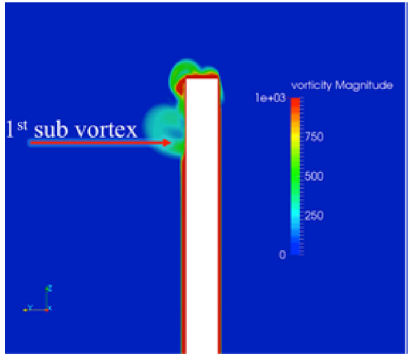

(a) FR D1

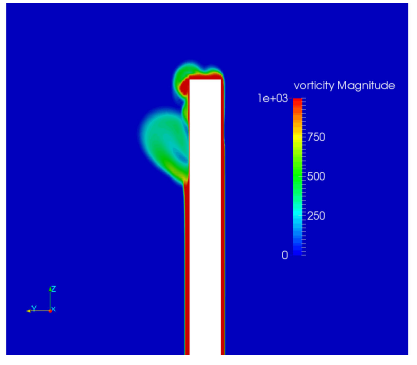

(b) FR D2

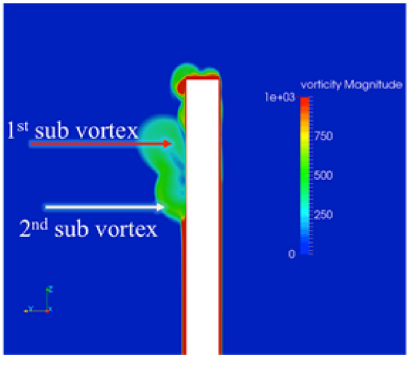

(c) FR D3

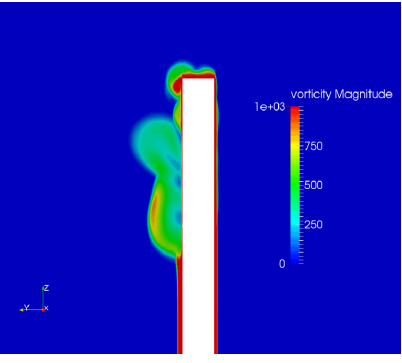

(d) FR D4

Fig. 18. Vorticity magnitude contour at $x / c=-0.4$.

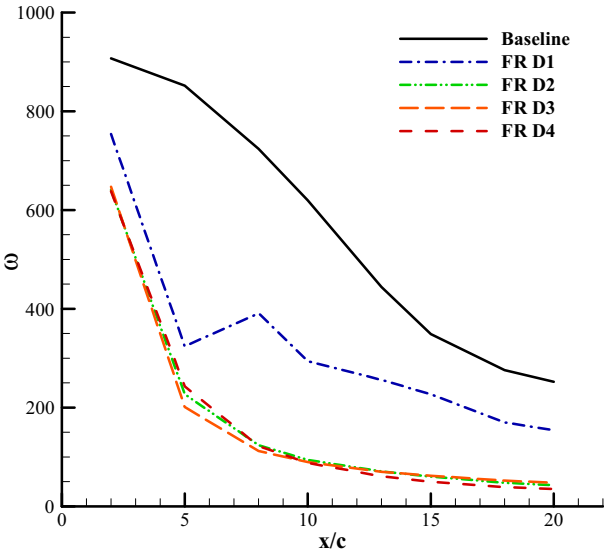

Fig. 19. Peak vorticity magnitude with chip depth.

\subsection{Effects of chip depth}

To evaluate the effects of the chip depth, the depth is varied at each location. For the MID chip and RE chip, chip depth is varied from D2 to D4. For the FR chip, which is the best chip shape for vortex dispersion, the chip depth is varied from D1 to D4.

\subsubsection{Vortex formation}

The vorticity magnitude contours of the FR chips at $x / c=-0.4$, right after the chip location, are presented in Fig. 18. As can be seen from Fig. 18(a) and (b), the FR D1 and FR D2 shapes generate only one sub-vortex, while two sub-vortices are generated for FR D3 and FR D4.

For FR D1, which has the shallowest depth, a weak and small sub-vortex is produced. Except for FR D1, the strengths and shapes of the first sub-vortex are similar for all of the cases.

\subsubsection{Peak vorticity magnitude}

Figure 19 compares the evolution of the vorticity magnitude for the FR chip with different chip depths. The reduction rates of vorticity magnitude increase as depth increases. The reduction rate changes from $38.8 \%$ for FR D1 up to $86.1 \%$ for FR D4. Except for FR D1, the reduction rate is almost similar. This is because of the weak first sub-vortex of FR D1 resulting from insufficient chip depth. This means that the process to attenuate the primary vortex using the first sub-vortex cannot occur. Through this, it is known that counter-rotating the first sub-vortex plays a key role in reducing the wingtip vortex strength.

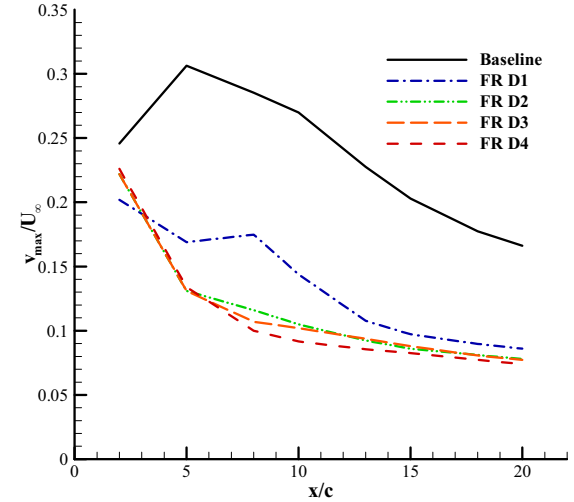

Fig. 20. Peak swirl velocity with chip depth.

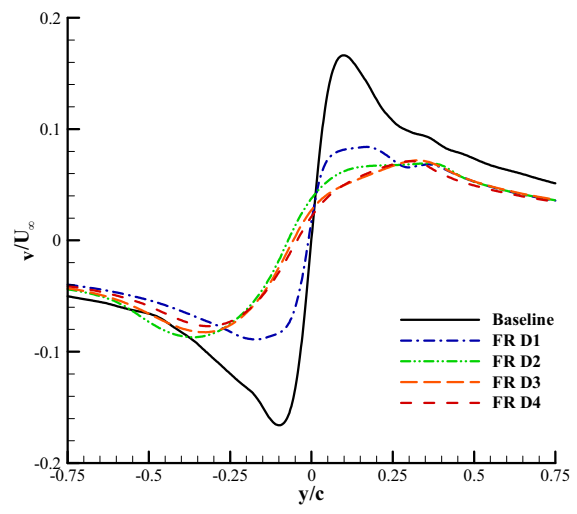

Fig. 21. Swirl velocity distribution with chip depth at $x / c=20$.

\subsubsection{Velocity distributions}

Figure 20 compares the evolution of the swirl velocity for the baseline shape and FR chips, which shows almost the same trend as in Fig. 18. The peak swirl velocity is decreased by $48.2 \%$ in FR D1 and FR D4 provided the highest reduction, $55.4 \%$.

The effects of FR chips on the swirl velocity distribution at $x / c=20$ are shown in Fig. 21. FR D1 has the smallest core radius; it increases by $50 \%$ compared to the baseline shape. FR D2, FR D3 and FR D4 give almost the same swirl velocity distribution. The core radius of FR D2-FR D4 chips increases by nearly $250 \%$ compared to the baseline.

The axial velocities for the FR chips at $x / c=20$ are compared to the baseline shape in Fig. 22. The shapes of axial velocity distributions look alike from FR D1 to FR D3. 
These three chips have a single peak value similar to the baseline shape. Only FR D4 has two peaks. Furthermore, when the chip depth increases from FR D1 to FR D4, the axial velocity deficit grows. The axial velocity deficits are $16.7 \%, 20.5 \%, 21.6 \%$, and $22 \%$ for each FR chip respectively. Thus, it is believed that chipped shapes that are deeper will be more dissipative as the result of radial transport of the angular momentum.

\subsection{Aerodynamic characteristics}

To evaluate the aerodynamic performance of each wing, aerodynamic coefficients and peak-to-peak swirl velocity $\Delta v_{p p}$ at $x / c=20$ are examined. The changes in aerodynamic characteristics and swirl velocity compared to the baseline are defined as Eqs. (1)-(3). All of parameters are non-dimensional, with reduced areas resulting from the chip. Compared to the baseline wing, the lift and swirl velocity always decrease and drag always increases.

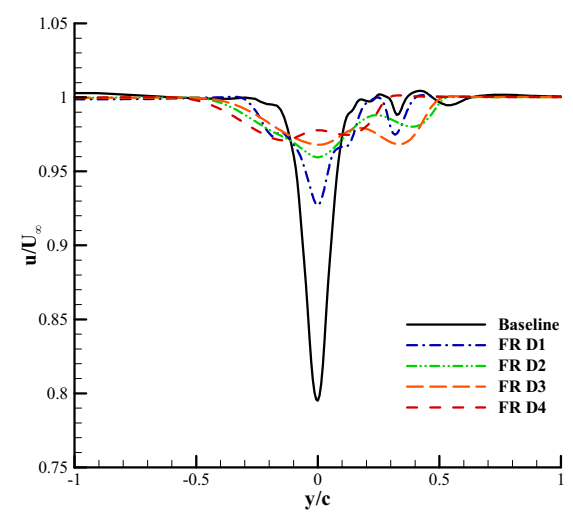

Fig. 22. Axial velocity distribution with chip depth at $x / c=20$.

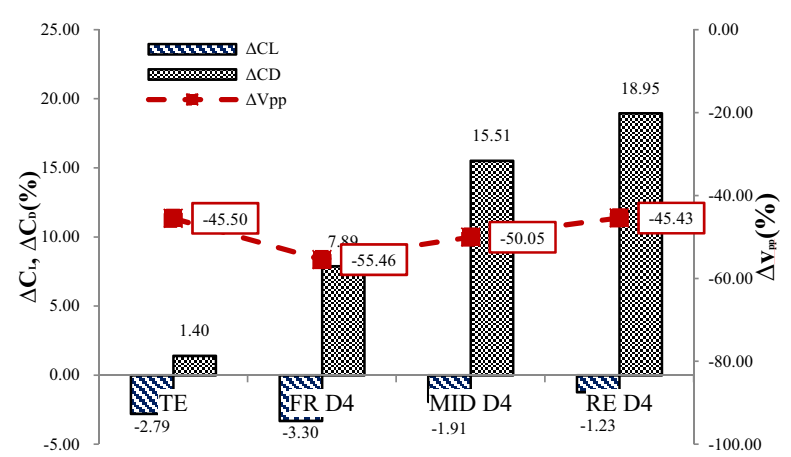

(a) Location variation

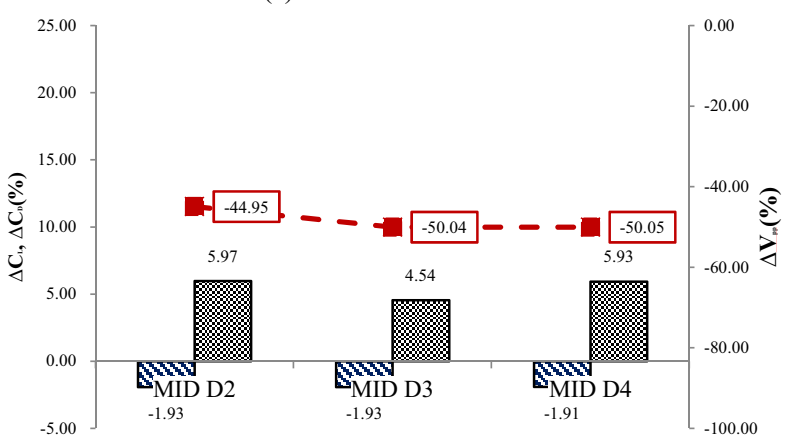

(c) MID variation

$$
\begin{aligned}
& \Delta C_{L}(\%)=\frac{C_{L, \text { chip }}-C_{L, \text { baseline }}}{C_{L, \text { baseline }}} \times 100 \\
& \Delta C_{D}(\%)=\frac{C_{D, \text { chip }}-C_{D, \text { baseline }}}{C_{D, \text { baseline }}} \times 100 \\
& \Delta v_{p p}(\%)=\frac{v_{p p, \text { chip }}-v_{p p, \text { baseline }}}{v_{p p, \text { baseline }}} \times 100
\end{aligned}
$$

The aerodynamic characteristics are shown in the bar chart. The $\Delta v_{p p}$ is shown as the red line.

Figure 23(a) shows the effects of chip location. Figure 23(b)-(d) compares the effects of chip depth at each location. From these results, it can be seen that wingtip vortex attenuation is clearly related to the aerodynamic characteristics of wingtip shapes. All of the tip-chipped shapes show increased drag when compared to the TE-chipped shape.

As shown in Fig. 23(a), a larger value of $\Delta v_{p p}$ can be obtained by moving the chip location from RE to FR, which implies more vortex alleviation. Additionally, with more vortex alleviation, as can be expected, higher drag and lower lifting forces are the result. Moving the chip from RE to FR, the vortex dissipation rate and drag increase by $10 \%$ and $17.3 \%$, respectively.

From Fig. 23(a), FR D4 shows the highest vortex reduction rate. However, FR D4 provides excessive drag. From Fig. 23(a), the vortex gap of the dissipation rate is almost constant from RE to FR, while the drag increase from MID to RE is smaller than that from MID to FR. Thus, the optimum chip shape that has low drag growth and a high vortex dissipation rate would exist between the RE and MID chips.

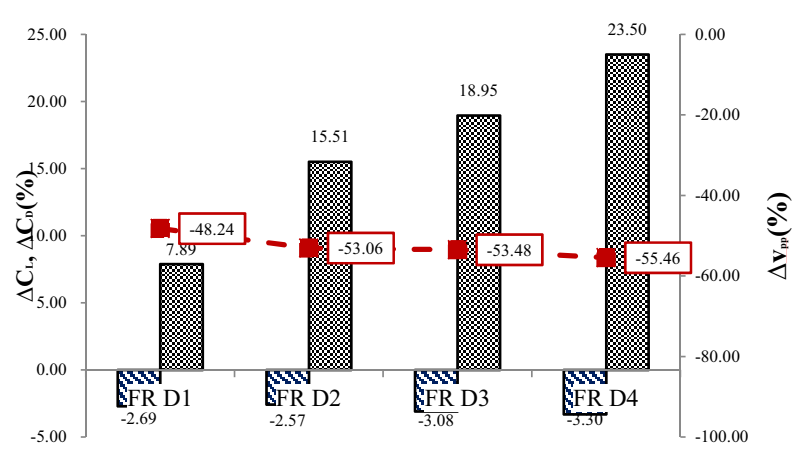

(b) FR variation

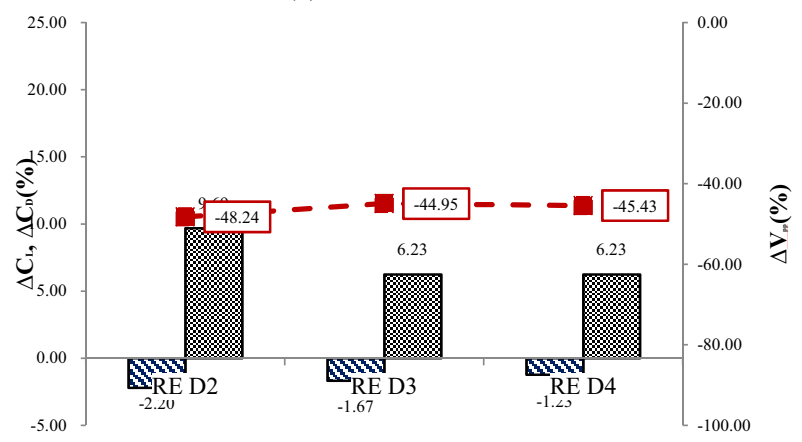

(d) RE variation

Fig. 23. Differences in aerodynamic characteristics and swirl velocity. 

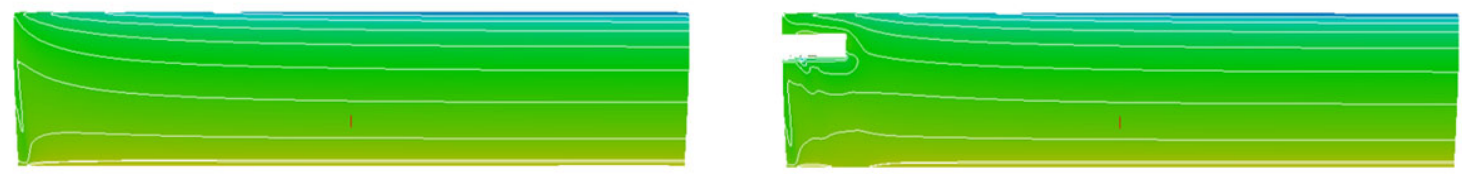

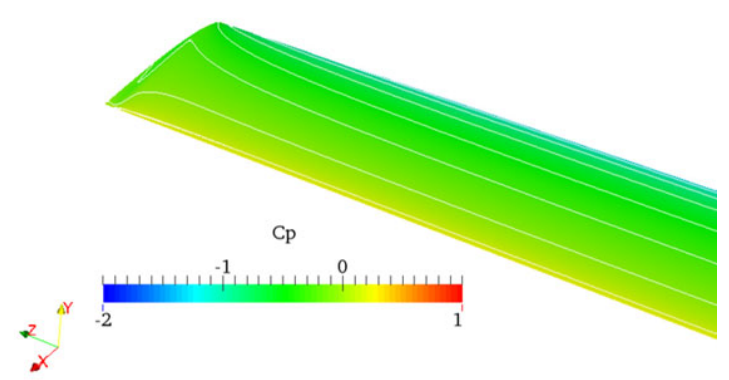

(a) Baseline wing

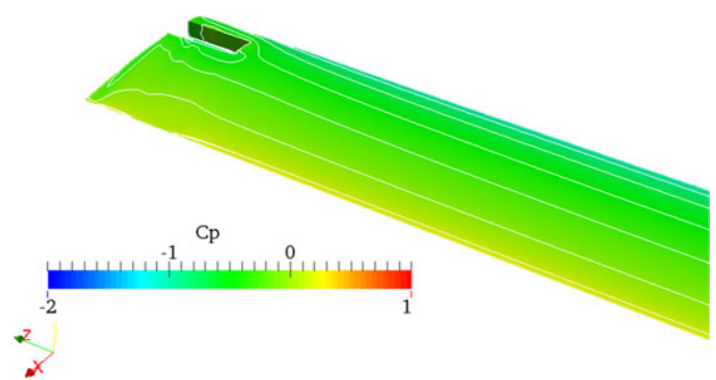

(b) FR D4

Fig. 24. Surface pressure distribution.

From Fig. 23(b)-(d), FR D4 is the best for vortex alleviation as previously explained. From Fig. 23(b), the dissipation rate of vortices is similar in FR D2 and FR D3. The dissipation slope is the steepest between FR D1 and FR D2. Therefore, the optimum depth is expected to be between 0.1 chord and 0.2 chord. Figure 23(c) shows, as the depth of the chip increases by 0.1 chord, drag increase by $1 \%$. The dissipation rate of vortex intensity increase by $6 \%$ from MID D2 to MID D3, but MID D3 and MID D4 are similar. From Fig. 23(d), the drag reduction rate and vortex dissipation rate for all RE chips is almost constant. Therefore, the location that has low drag growth and high vortex dissipation rate is between MID and RE (Fig. 23(a)), and the depth of the chip is between 0.2 and 0.3 chord (Fig. 23(c)).

As can be seen in Fig. 23, the rate of lift reduction is relatively small compared to the rate of vortex reduction. At the chipped tip shape, there is a slight reduction in the total circulation due to chip, as is suggested by the Kutta-Joukowski theorem, which states that 2D lift is directly proportional to the circulation. However, the chip area is relatively negligible compared to the total area of the wing (1.3\% of total wing area). As is shown in Fig. 24, surface pressure distribution is not altered significantly, except in the chipped area, this being due to the existence of chip. That is, the chip generates the sub-vortex with relatively little loss of total lift. The lift in Fig. 23 is calculated from the pressure on the wing surface, and the swirl velocity is obtained at 20 chords behind the wing. It is clearly summarized in Fig. 23 that there is a slight decrease in lift due to the chip, but the counterrotating sub-vortex tends to accelerate dissipation of the primary vortex in the wake.

Figure 25 shows these design points for conventional aircraft and rotor blades. FR D4 indicates the highest vortex reduction rate and the highest increase in drag. However, considering operation on the runway, where the influence of vortex is dominant, increasing drag when landing is not a serious issue. Additionally, aerodynamic losses when cruising can be minimized by applying ideas ${ }^{25)}$ for $L / D$ preserva-

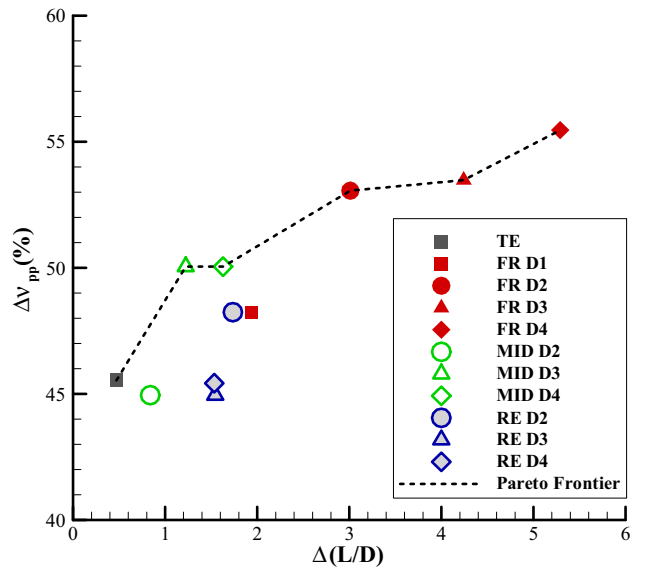

Fig. 25. Trade-off relationship between the increment of $\Delta C_{D}$ and the decrement of $v_{p p}$ at $x / c=20$.

tion. Thus, FR D4 can be recommended for conventional aircraft. Helicopter rotors generate a more highly complex flow than conventional aircraft. Therefore, to investigate the effects of reducing the BVI using a chipped shape, rotor analysis is essential. For that analysis, MID D3, which results in a minimum increase in drag and a vortex reduction rate above $50 \%$, would generally be worth implementing.

\section{Conclusion}

In an effort to increase the efficiency of airport operation, as well as to decrease BVI noise, a chipped tip shape is designed to alleviate wingtip vortex strength. This study suggests utilizing a wing that has a chip at the wingtip to accelerate the aerodynamic interference between the tip vortices. Specifically, it is intended to show the difference in the vortex interference mechanism between TE-chipped wing and tip-chipped wings. In addition, the study clarifies the effects of chip location and chip depth on the tip-chipped wing. Vortex parameters such as maximum vorticity magnitude, swirl velocity and axial velocity were investigated. The relation 
between vortex intensity dissipation rate and aerodynamic drag was carefully examined. Consequently, the following conclusions were reached;

1) The vortex dissipation rate of the tip chip is higher than that of the TE chip. The tip chip interferes with the generation of wingtip vortex, weakening the primary vortex more efficiently. In addition, the counter-rotating sub-vortex tends to accelerate dissipation of the primary vortex in the wake.

2) For the same chip area, the vortex reduction rate increases as the chip is located closer to the leading-edge. If the chip is located close to the leading-edge, the chip experiences larger differences in pressure. The stronger sub-vortices caused by larger pressure differences have the effect of accelerating the vortex alleviation rate. Simultaneously, drag is proportional to the dissipation rate of vortex intensity.

3) The case of FR D4 provided greater vortex dissipation than all of the other cases when comparing axial velocity distributions at 20 chord downstream.

4) As the chip depth increases, the vortex reduction rate and drag growth tend to increase. The aerodynamic effects of chip depth are more sensitive when the chip is closer to the leading-edge. The reduction rate changes from $48.2 \%$ for FR D1 up to $55.4 \%$ for FR D4. The $\Delta C_{D}$ changes from $7.5 \%$ for FR D1 to $23.5 \%$ for FR D4.

5) The vortex intensity dissipation rate and the increases in $\Delta C_{L / D}$ have a proportional relationship. However, the reduction in $\Delta C_{L / D}$ is not a linear function of $\Delta v_{p p}$. FR D4 is the best for vortex alleviation, but also provides the highest drag, which is recommended for the landing approach of conventional aircraft. MID D3 results in the minimum increase in drag with a vortex reduction rate above $50 \%$, which is suitable for rotor blade shapes.

\section{Acknowledgments}

This research was supported by the Basic Science Research Program of the National Research Foundation of Korea (NRF) and funded by the Ministry of Science, ICT and future Planning (NRF-2014R1A2A1A10054537).

\section{References}

1) Rossow, V. J.: Wake-vortex Separation Distances when Flight-path Corridors are Constrained, AIAA J., 33, 3 (1996), pp. 539-546.

2) Rossow, V. J.: Lift-generated Vortex Wakes of Subsonic Transport Aircraft, Progress in Aerospace Sciences, Vol. 35, 1999, pp. 507-660.

3) Tangler, J. L.: Schlieren and Noise Studies of Rotors in Forward Flight, 33rd American Helicopter Society Forum, Washington, DC, 1977.

4) Yu, Y. H., Gmelin, B., Splettstoesser, W., Philippe, J. J., and Prieur, J.: Reduction of Helicopter Blade-Vortex Interaction Noise by Active Rotor Control Technology, Progress in Aerospace Sciences, Vol. 33,
1997, pp. 647-687.

5) Rayleigh, L.: On the Dynamics of Revolving Fluids, Proc. R. Soc. London Ser. A, 93 (1917), pp. 148-154.

6) Batchelor, G. K.: Axial Flow in Trailing Line Vortex, J. Fluid Mech., 20 (1964), pp. 645-658.

7) Saffman, P. G.: Structure of Turbulent Line Vorties, Phys. Fluids, 16 (1973), pp. 1181.

8) Bleviss, Z. O.: Theoretical Analysis of Light Plane Landing and Takeoff Accidents Due to Encountering the Wakes of Large Airplane, Douglas Aircraft Co., Inc, Santa Monica, CA, 1954.

9) Rossow, V. J.: Convective Merging of Vortex Cores in Lift-Generated Wakes, J. Aircraft, 14 (1977), pp. 283-290.

10) Devenport, W. J., Rife, M. C., Liapis, S. I., and Follin, G. J.: The Structure and Development of a Wingtip Vortex, J. Fluid Mech., 312 (1996), pp. 67-106.

11) Chow, J. S., Zilliac, G. G., and Bradshaw, P.: Mean and Turbulence Measurements in the Near Field of a Wingtip Vortex, AIAA J., 35 (1997), pp. 1561-1567.

12) Rossow, V. J.: Inviscid Modeling of Aircraft Trailing Vortices, Proceedings of NASA Symposium on Wake Vortex Minimization, 1977, pp. 9-60.

13) Mineck, R. E.: Assessment of Potential Aerodynamic Benefits from Spanwise Blowing at the Wingtip, Ph.D. Thesis, The George Washington University, 1992.

14) Patterson, Jr. J. C.: Wingtip Vortex Turbine, 4917332, US.

15) Rossow, V. J.: Effect of Wing Fins on Lift-Generated Wakes, J. Aircraft, 15 (1978), pp. 160-167.

16) Claude, G. M. and John, K. E.: Wake Vortex Alleviation Using Rapidly Actuated Segmented Gurney Flaps, AIAA J., 45 (2007), pp. 1874-1884.

17) Nelson, C. T. and Rediniois, O. K.: Ac Active Flap Deployment System for Blade-Disturbance Interaction Alleviation, J. Engineering, 126 (2005), pp. 1006-1014.

18) Han, Y. O. and Lesihman, J. G.: Investigation of Helicopter RotorBlade-Tip-Vortex Alleviation Using a Slotted Tip, AIAA J., 42 (2004), pp. 524-525.

19) Landgrebe, A. J. and Bellinger, E. De.: Experimental Investigation of Model Variable-Geometry and Ogee Tip Rotors, NASA Report, CR2275, 1974.

20) Jin, J. H., Oh, S. J., and Yee, K. J.: Numerical Simulation of Trailed Vortex Alleviation through Chipped Wingtip Shape, J. Mech. Sci. Technol., 28 (2014), pp. 3605-3615.

21) Shin, T. H., Liou, W. W., Shabbir, A., Yang, Z., and Zhu, J.: A New k- $\epsilon$ Eddy Viscosity Model for Turbulent Flow High Reynolds Number, Comput. Fluids, 24 (1995), pp. 227-238.

22) Davis, P. L., Rinehimer, A. T., and Uddin, M.: A Comparison of RANS-Based Turbulence Modeling for Flow over a Wall-Mounted Square Cylinder, Proc. 20th Annual Conference of the CFD Society of Canada, 2012.

23) Kim, T. W.: The Development of a GUI Environment Automatic Program for Rotor Blade Airfoil Performance Analysis, MS Thesis, Pusan National University, 2009.

24) Uberoi, M. S.: Mechanisms of Decay of Laminar and Turbulent Vortices, J. Fluid Mech., 92 (1979), pp. 241-255.

25) Roh, N. H., Yee, K. J., and Oh, S. J.: Numerical Study on Vortex Alleviation of Chipped Wings, 2014 Asia-Pacific International Symposium on Aerospace Technology, Shanghai, China, APISAT 2014, 2014.

S. Saito

Associate Editor 